\title{
A Composite PID Fuzzy Control Method for the Transformer Cooling System
}

\author{
Liu Bing \\ State Grid Songyuan Power Supply Company of Jilin \\ Electric Power Co., Ltd \\ Jinlin, China \\ 1_bin@163.com
}

\author{
Han Zhaoting \\ State Grid Songyuan Power Supply Company of Jilin \\ Electric Power Co., Ltd \\ Jinlin, China \\ ZT-Han@hotmail.com
}

\begin{abstract}
PID control algorithm is used for the control of almost all loops in the process industries, and is also the basis for many advanced control algorithms and strategies. Standard methods for tuning loops and criteria for judging the loop tuning have been used for many years, but should be reevaluated for use on modern digital control systems. For the shortcomings of traditional PID control, a composite PID fuzzy control method for transformer cooling system is proposed. The method combines fuzzy control and PID control, which form composite fuzzy PID control method based on conventional PID control. With obtain temperature from the thermometer, it can find input error $E$ and error change EC, and quantify the fuzzy, compute fuzzy control table, check the table to obtain fuzzy control parameter, find the actual PID input control parameter. Finally it find the output control parameter to the inverter. The proposed method uses PID proportional control to improve the dynamic response rate, and uses PID integral control to eliminate the steady state error, which improves the steadystate performance of fuzzy control, thus further improving energy savings transformer cooling system operation.
\end{abstract}

Keywords- composite PID fuzzy control; transformer cooling system; fuzzy control parameter

\section{INTRODUCTION}

Transformer is important equipment of power plants and substations. With the continuous expansion and improvement of the voltage level of the power scale systems, the current total capacity of the power system transformer has reached 9-10 times the total power generation capacity. Thus, secure, stable operation of the transformer is directly related to the reliability of the power supply industry, agriculture and civil. According to the study, the transformer winding temperature is increased by $6^{\circ} \mathrm{C}$, the life of transformer will be shortened by half, so the real-time monitoring and implementation of the transformer cooling is extremely necessary[1-3].

At present, at home and abroad main transformer cooling methods of power system to run large and medium-sized are the following [4-6] :

(1) Natural air cooling mode;

(2) Forced oil circulation air cooling mode;

(3) Forced oil circulation water cooling mode;

(4) Forced air cooling oil circulation-oriented mode;

(5) Low boiling point medium evaporative cooling mode.
Small-capacity transformers generally use natural air cooling mode. Large-capacity transformers of substations generally use forced air cooling oil circulation. For power plants, due to adequate water, forced oil circulation water cooling and forced oil circulation air cooling methods use these modes [7]. Currently there are many running large and medium-sized transformers forced oil circulation air cooling methods. Low boiling point medium evaporative cooling of large and medium-sized transformers made by Shenzhen Auto Electric Equipment Co. Ltd had passed the qualification test by Wuhan High Voltage Research Institute transformer inspection stations, but there is no specific application information [8-9].

Large and medium-sized transformers of foreign power system running were more than $80 \%$ using forced oil circulation air cooling. Early commissioning of products commonly used contact relay logic control transformer cooler simple switching, which is controlled by temperature mechanical relay contacts open and close to drive AC contactor to control the Group's coolers input and exit. The features of this operating mode are electrical wiring complexity, the device failure rate, controlled low accuracy. When the temperature changes near the setting value, control devices are often frequent switching phenomenon [10]. When the cooler had been working, the cooling fans who are working are all at frequency $(50 \mathrm{~Hz})$, which runs the state that increased electricity of the plant, while the life of rotating equipment fans, motors has also been restrictions.

With the development of power electronics technology, in order to overcome the shortcomings of the high failure rate of mechanical contacts, engineering and technical personnel at home and abroad in recent years use solidstate relays and other non-contact devices to replace conventional mechanical contacts, exchanges and contacts, which achieved better test results. The failure rate of coolers reduced [11]. Meanwhile, with the development of computer technology and PLC, in foreign countries computer or PLC are used for large and medium-sized transformer cooling system to implement optimal control. In recent years, the domestic manufacturers and research institutes are also relevant for large and medium-sized transformer cooling system to control device for continuous improvement. For example, the "frequency transformer cooled energy-saving devices" using PLC and inverter the implementation of the transformer cooler 
control achieved good results developed by one company of Northeast Institute of Electric Power.

Currently, the configuration of large power transformer cooling device is based on the size of the transformer capacity, configuration, and multiple air-cooled oil circulation cooling devices. Each air-cooled oil circulation cooling device consists of an oil pump and 3-4 fan components, which is used to meet a variety of operating conditions of the transformer, generally require 1 spare cooler (when running cooler failure can be automatically put into operation), an auxiliary cooler (or load current transformer top oil temperature higher than the set automatically put into operation the threshold value), all the rest of the cooler into operation [12-16]. The traditional chiller control system has drawbacks, the safety and reliability of intelligent power system has failed to meet the growing demand control, so the chiller control system transformation imperative.

\section{TRADITIONAL PID CONTROL THEORY}

The $\mathrm{P}$ stands for proportional control, I for integral control and D for derivative control. This is also what is called a three term controller. The basic function of a controller is to execute an algorithm (electronic controller) based on the control engineer's input (tuning constants), the operators desired operating value (set point) and the current plant process value. In most cases, the requirement is for the controller to act so that the process value is as close to the set point as possible. In a basic process control loop, the control engineer utilizes the PID algorithms to achieve this.

The PID control algorithm is used for the control of almost all loops in the process industries, and is also the basis for many advanced control algorithms and strategies. In order for control loops to work properly, the PID loop must be properly tuned. Standard methods for tuning loops and criteria for judging the loop tuning have been used for many years, but should be reevaluated for use on modern digital control systems. While the basic algorithm has been unchanged for many years and is used in all distributed control systems, the actual digital implementation of the algorithm has changed and differs from one system to another.

The PID (proportional, integration, differentiation) controller is the earliest practical controller for nearly a hundred years of history, which is still the most widely used industrial controllers. PID controller is simple to understand and use, without a prerequisite for accurate system model, which become the most widely used controller. At present, PID control and its controller or intelligent PID controller (meter) has lots of products have been in engineering practice a wide range of applications, there are a variety of PID controllers, the major companies have developed with PID parameter self-tuning features intelligent regulator (intelligent regulator), which automatically adjusts the PID controller parameters are adjusted through intelligent or self-correction, adaptive algorithm. There PID control is realized by the pressure, temperature, and flow, level controller, PID control function enables the programmable controller, as well as enables PC system with PID control and so on.

If differential action is increased, the speed of the system response is increase, so that the overshoot is reduced, the stability is increased. But at the same time the disturbance of system is sensitive, interference suppression capacity is diminished. If the differential is large, the process of response can be brake much earlier, and extended over time.

If the differential action is reduced, adjustment process will lag, so that the overshoot is increased, the speed of system response is slow, stability deteriorates. Therefore, for time-varying and uncertain systems, such as thermal processes, the time constant of the controlled object should be adapted to random change.

According to long-term operational experience in the early response process, the derivative action is appropriate to increase which can even avoid overshoot. At response process in mid, because D is the very sensitive, D should be smaller and remains unchanged. In the later adjustment process, D is smaller again, thereby the effect of the braking process is weakened and the ability to inhibit the disturbance is increased. At the initial adjustment stage, D is larger, the adjustment time is growth compensated. Integral and differential adjustment is usually used in conjunction with proportional control action to achieve different control performance.

\section{THE COMPOSITE FUZZY PID CONTROL METHOD}

The detailed description of composite fuzzy PID control method is show: Obtain temperature from the thermometer; find input error E and error change EC, and to quantify the fuzzy; compute fuzzy control table, check the table to obtain fuzzy control parameter; find the actual control parameter $K_{P}, K_{I}, K_{D}$, which is PID input parameter; finally find the control parameter $y$, output to the inverter. The block diagram of composite fuzzy PID control method is shown in Fig .1.

First, obtain temperature through the thermometer, specifically temperature is measured through the thermometer in real time transformer oil temperature. Find the input error $E$ and error change $E C$, then quantify and fuzzy. Specifically compared the oil temperature realtime measured with setting stored in advance to obtain $e$ and $e c$. in the control system of transformer the basic domain of oil temperature error e:

$$
\left[-\frac{T_{\max }-T_{\min }}{2}, \frac{T_{\max }-T_{\min }}{2}\right]
$$

Where $T_{\max }$ is the maximum safe value of transformer oil temperature. $T_{\min }$ is the minimum safe value of transformer oil temperature. The maximum value of the test results by fundamental changes ec(the oil temperature deviation change rate) is $0.5^{\circ} \mathrm{C}$, therefore, the range of the basic domain of $e c$ is $\left[-\frac{1}{2}, \frac{1}{2}\right]$. 


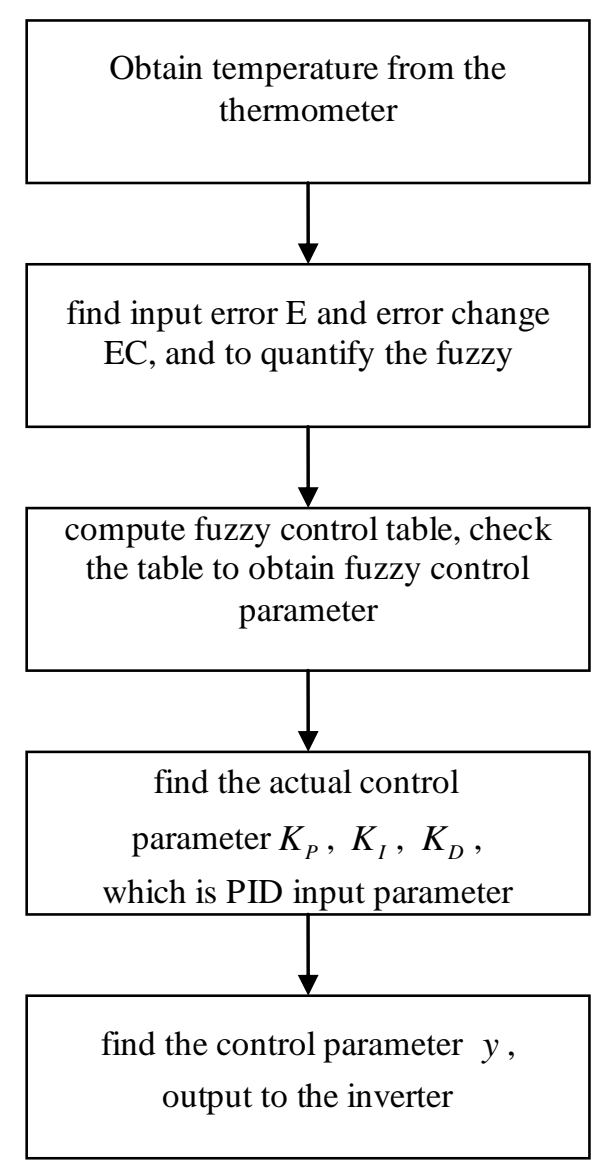

Figure1. The block diagram of composite fuzzy PID control method

Fuzzy controller design needs quantifying the precise amount of input multiplied by the quantization factor, the fuzzy controller inputs that fall within the set of fuzzy domain, and where the fuzzy value of $e$ and $e c$ are respectively $E$ and $E C$. Because as long as the appropriate scaling factor is selected, the corresponding fuzzy domain can be obtained, which does not affect the fuzzy controller design. In the design of the controller, select the quantization factor $k_{e}=\frac{2}{5}, k_{e c}=12$.

The fuzzy control table is calculated, and checks the table to obtain the fuzzy control volume. Specifically the domain of fuzzy sets based on the deviation $e$ is taken $\{-6$, $-5,-4,-3,-2,-1,0,+1,+2,+3,+4,+5,+6\}$ language variable value $\{$ negative big, negative middle, negative small, zero, positive small, positive middle, positive big , abbreviated as $\{\mathrm{NB}, \mathrm{NM}, \mathrm{NS}, \mathrm{O}, \mathrm{PS}, \mathrm{PM}, \mathrm{PB}\}$ the fuzzy domain of deviation of the change rate $e c$ are $\{-6,-5,-4$, $3,-2,-1,0,+1,+2,+3,+4,+5,+6\}$ language variable values are $\{\mathrm{NB}, \mathrm{NM}, \mathrm{NS}, \mathrm{O}, \mathrm{PS}, \mathrm{PM}, \mathrm{PB}\}$.

In the PID fuzzy controller, fuzzy set of input variables $e$ and $e c$ are (NB, NM, NS, O, PS, PM, PB), which is to determine the membership of its inputs by summing the experience of the operator method degrees. Fuzzy variables control table can be obtained by check the fuzzy control table.

Find the adjust amount $\Delta K_{P}, \Delta K_{I}, \Delta K_{D}$ of the amount of actual control $K_{P}, K_{I}, K_{D}$, and enter the amount as the PID; Specifically the fuzzy controller input temperature deviation $e$ and the temperature deviation rate ec, $\Delta K_{P}, \Delta K_{I}, \Delta K_{D}$ are 3 adjust value output of the PID controller. Take the input and output linguistic variables fuzzy subset $\{\mathrm{NB}, \mathrm{NM}, \mathrm{NS}, \mathrm{O}, \mathrm{PS}, \mathrm{PM}, \mathrm{PB}\}$, the input and output are quantified to 7 levels, whose fuzzy domain are $\{-3,-2,-1,0,+1,+2,+3\}$, All fuzzy subset select the triangular distribution membership function. Define $K_{P}, K_{I}, K_{D}$ adjust equation.

$$
\left\{\begin{array}{l}
K_{P}=K_{P 0}-\Delta K_{P} \\
K_{I}=K_{I 0}-\Delta K_{I} \\
K_{D}=K_{D 0}-\Delta K_{D}
\end{array}\right.
$$

Where, $K_{P 0}, K_{I 0}, K_{D 0}$ are respectively the initialization parameters of $K_{P}, K_{I}, K_{D}$, when real-time operation, the output response value of the engine cooling system calculate the deviation $e$ and the deviation change rate $e c$, then fuzzyed to obtain $E$ and $E C$, then query the fuzzy variable table query, 3 adjustment amount $\Delta K_{P}$, $\Delta K_{I}, \Delta K_{D}$ can be obtained, then the adjustment of the controller parameters is completed.[9-16]

Find the amount of control $y$, and output to the inverter. Specifically the output $y$ can be obtained from the following equation 2 .

$$
\begin{aligned}
y & =K_{p}\left\{e(k)+\frac{T_{0}}{T_{I}} \sum_{i=0}^{k} e(i)+\frac{T_{D}}{T_{0}}[e(k)-e(k-1)]\right\} \\
& =K_{p} e(k)+K_{I} \sum_{i}^{k} e(i)+K_{D}[e(k)-e(k-1)]
\end{aligned}
$$

Where $T_{0}$ is the sampling period, $T_{I}$ is the time constant of integral $I, T_{D}$ is the time constant of differential D.

The distribution of the membership function of $K_{P}$, $K_{I}, K_{D}$. The measured deviation transformer top oil temperature and the deviation e, the rate of change ec is defined as input, corresponding decision by the fuzzy reasoning based on input channel, the 3 parameters $K_{P}$, $K_{I}, K_{D}$ of PID regulator are adjusted in real-time, precisely controlled speed of cooling fan, so that the heat and cool of the transformer can achieve balance in realtime.

Based on embodiment of the test obtained in scene, when the conventional PID controller is used, in the temperature difference PID control parameters $K_{P}, K_{I}$, $K_{D}$ are real-time adjusted according to the calculation instructions. Finally the range of the 3 control parameters are respect $[20,70],[3,15],[0,10]$. Therefore, the basic domain of 3 control parameters $K_{P}, K_{I}, K_{D}$ of PID controller are $[0,100],[0,20],[0,10]$, the scaling factor was $\Delta K_{P}=\frac{100}{3}, \Delta K_{I}=\frac{20}{3}, \Delta K_{D}=\frac{10}{3}$, which are 
the scaling factors of output control value $K_{P}, K_{I}, K_{D}$ is selected, fuzzy domain are $\{-3,-2,-1,0,1,2,3\}$, language variable values are (NB, NM, NS, O, PS, PM, $\mathrm{PB})$. The distribution of the membership function of $K_{P}$, $K_{I}, K_{D}$ are shown in Fig .2.

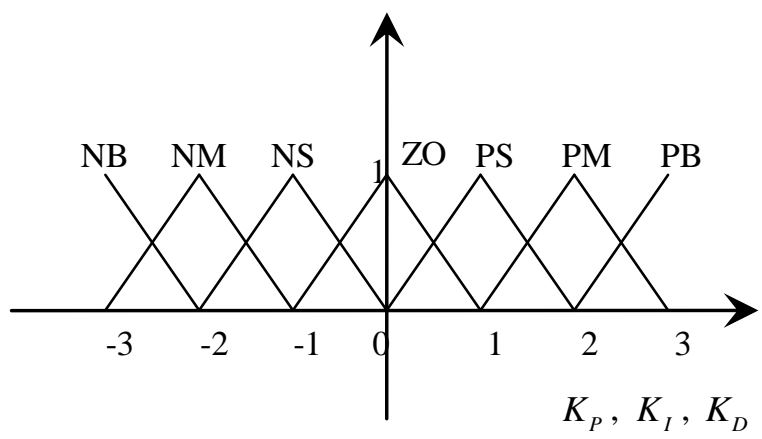

Figure 2. The distribution of the membership function of $K_{P}, K_{I}$,

$$
K_{D} \text {. }
$$

\section{CONCLUSION}

For the shortcomings of traditional PID control, a composite PID fuzzy control method used to transformer cooling system is propose, the method combines fuzzy control and PID control, which form composite fuzzy PID control method based on conventional PID control, The proposed method uses PID proportional control to improve the dynamic response rate, uses PID integral control to eliminate the steady state error, which improves the steady-state performance of fuzzy control, thus further improving energy savings transformer cooling system operation

\section{ACKNOWLEDGMENT}

This work was supported in part by the Key Project of the National Nature Science Foundation of China (No. 60534020), This work was supported by a grant from the National High Technology Research and Development Program of China (863 Program) (No. 2012AA040104), A Project Supported by Scientific Research Fund of Jilin Provincial Education (20120268). A Project Supported by Scientific and Technological Planning Project of Jilin Province (20120332). A Project Supported by Scientific and Technological Planning Project of Jilin Province (20100565).

\section{REFERENCES}

[1] G.Chen "Conventional and Fuzzy PID Controllers:an Overview" Int J of Intelligent Control\&Systems, vol.1(2), pp: 235-246, 1996.

[2] F. Jiang, Z.Gao. "An application of nonlinear PID control to a class of truck ABS problems". Proceedings of the 40th IEEE Conference on Decision and Control, vol. 1,pp: 516-521,2001.

[3] F.Georg, F.Gérard, Y.C. Gissinge. "Fuzzy Logic Continuous and Quantizing Control of an ABS Braking System”. SAE Paper No. 940830, 1994.

[4] Mark A key.Development of Fuzzy Logic ABS Control for Commercial Trucks.SAE Paper No.952673, 2005.
[5] G.Kokes,"Tarunraj Singh.Adaptive Fuzzy Logic Control Uf An Anti-Lock Braking System". International Conference on Control Applications, vol. 8,pp: 646-651.2009

[6] G.Marjan.”Decomposed fuzzy proportional-integral-derivative controllers". Applied Soft Computing,vol 1(3),pp: 201-214.2011

[7] J Xu, C,Hang C Liu. "Parallel structure and tuning of a fuzzy PID controller”. Automatica, vol 36(3),pp: 673-684.2000.

[8] J.H.Kim,S.J.Oh.”A fuzzy PID controller for nonlinear and uncertain systems".Soft Computing, vol 4,pp. 123-129,2010..

[9] D. XiaoGanguan, L. HanXiong. “A Simple Tuning Method for Fuzzy PID Control”. IEEE International Conference on Fuzzy Systems, pp: 271-275.2008.

[10] W.ChiaJu,L.TsongLi."Auto-Tuning Fuzzy PID Control of a Pendubot System". Proceedings of International Conference on Mechatronics, pp: 1-6.2007

[11] P. Ananthababu, B. Amarendra Reddy,"haran.Design of Fuzzy PI+D and Fuzzy PID Controllers Using Gaussian Input Fuzzy

[12] Sets".Second International Conference on Emerging Trends in Engineering and Technology,pp:957-961.2009

[13] D.Sandhya, B.Amarendra Reddy, A.Prof.K . "Fuzzy I-PD and Fuzzy PID Control Of Non-linear Systems". Control Automation Comunication And Energy Conservation,vol.4,pp: 1-6.2009

[14] K.S.Tang, F. M Kim,"An optimal fuzzy PID controller," Industrial Electronics, vol.48, pp: 757-765,2001

[15] Y.Yuanhui, Y.Wailing,W.Mingchun,Y.Qiwen,X.Yuncan,"A New Type of Adaptive Fuzzy PID Controller," Intelligent Control and Automation (WCICA), 8th World Congress on, pp 53065310,2010

[16] D.Chengsong, C. Lijun, "Self Adaptive Fuzzy PID Controller for Water Supply System", International Conference on Measuring Technology and Mechatronics Automation, pp, 311-314,2010

[17] E.Pathmanathan, R.Ibrahim, "Development and Implementation of Fuzzy Logic Controller for Flow Control Application," Intelligent and Advanced Systems (ICIAS), International Conference on, vol., 16, pp. 15-17,2010 